\title{
The effects of flicker adaptation upon temporal contrast enhancement
}

\author{
FREDERICK L. KITTERLE and BETTINA L. BEARD \\ University of Toledo, Toledo, Ohio
}

\begin{abstract}
Temporal contrast enhancement refers to the finding that intermediato-duration, low-spatialfrequency gratings are perceived to have a greater contrast than long-duration gratings of similar spatial frequency. Kitterle and Corwin (1979) suggested that this effect reflects primarily activity in transient channels. To test this, 30 subjects were run in one of three groups consisting of either adaptation to a steady or flickering low-spatial-frequency grating or no adaptation. Temporal contrast enhancement was found for both steady and no-adaptation conditions. Flicker adaptation abolished temporal contrast enhancement. It was suggested that flicker adaptation may decrease the contribution of transient channels to perceived contrast and may cause processing of the test gratings to be switched to sustained channels. The implications of these results for understanding the coding of brightness are discussed.
\end{abstract}

Psychophysical studies have shown that a flickering grating or line can be set to threshold using a flicker or pattern-detection criterion (Keesey, 1972; Kulikowski \& Tolhurst, 1973; Tolhurst, 1973; van Nes, Koenderink, Nas, \& Bouman, 1967). At low spatial frequencies, the human visual system is more sensitive to flicker than to pattern, whereas at high spatial frequencies, the reverse is found. Kulikowski and Tolhurst (1973) have concluded that flicker and pattern perception are determined by functionally independent mechanisms. They have suggested that flicker detection is mediated by transient channels, which are more sensitive to low-spatial-frequency gratings temporally modulated at high rates, and that sustained channels, which are more sensitive to intermediate-to-high spatial frequencies temporally modulated at low rates, mediate pattern detection.

Burbeck (1981) has shown that these original findings resulted from the use of the method of adjustment. In this method, flicker thresholds are found to be lower than pattern thresholds because the flicker task requires less information and therefore is easier to detect than a pattern. Using a criterion-free method that requires the observer to distinguish the test stimulus from a standard differing in only one dimension (the spatial structure in the pattern experiments or temporal factors in the flicker experiments), Burbeck (1981) has shown that pattern sensitivity is equal to or greater than flicker sensitivity for all flickering gratings except those that have extremely low spatial frequency or moderate-to-high temporal frequencies. These results do not support the identification of flicker detection within transient mechanisms and pattern detection within sustained mechanisms,

The authors' mailing address is: Department of Psychology, University of Toledo, Toledo, Ohio 43606. whereas they do indicate that flicker and pattern detection are mediated by different physiological mechanisms. In addition, Green (1981a) has shown that the transient system can mediate perception for gratings up to $30 \mathrm{cycles} / \mathrm{deg}$. Thus, recent studies question the conclusion of Kulikowski and Tolhurst (1973) that flicker detection is mediated solely by transient channels and pattern detection by sustained channels.

Nevertheless, the existence of these two channels has been supported by masking studies (Green, 1981 b; Kitterle, Corwin, \& Berta, 1979; Mitov, Vassilev, \& Manahilov, 1981), by differences in adaptability to uniform field flicker (Green, 1981a), and by differences in contrast gain control (Burbeck \& Kelly, 1981). Other studies indicate that the limits of temporal summation are shorter for transient than for sustained channels (Legge, 1978; Nachmias, 1967) and that the latencies of transient channels are shorter than those of sustained channels (Breitmeyer, 1975). These two mechanisms also differ in their temporal impulse response. Transient channel responses are biphasic: they consist of an early excitatory component and a delayed inhibitory response (Kelly, 1971a, 1971b; Ueno, 1977; Watson \& Nachmias, 1977), whereas there does not appear to be any difference in the latency of the excitatory and inhibitory responses of sustained channels (Kitterle, 1979; Watson \& Nachmias, 1977).

Little is known about the role played by these channels at suprathreshold levels of illumination. Broca and Sulzer (1903) have shown that the perceived brightness of an intermediate-duration (50-130-msec) flash is greater than that of a long-duration flash of the same luminance. This phenomenon has been called the "Broca-Sulzer effect" or "temporal brightness enhancement." Several models that emphasize the role of neural response transients and delayed inhi- 
bition have been postulated to account for this effect (e.g., Adrian, 1928; Baumgardt \& Segal, 1947; Boynton, 1961).

Kitterle and Corwin (1979) tested the possibility that temporal brightness enhancement is mediated by transient channels by measuring changes in apparent contrast for sinusoidal gratings as a function of flash duration and grating spatial frequency. It was found that the function relating apparent contrast and duration varies with spatial frequency. Apparent contrast increased with duration at a rate inversely related to spatial frequency, a result consistent with the threshold summation data of Legge (1978). At longer durations, however, apparent contrast became independent of duration, and increased at a considerably slower rate for high spatial frequency targets. The changes in apparent contrast were more complex for the low-spatial-frequency targets. At longer durations, apparent contrast decreased and then became independent of duration. Thus, the apparent contrast of long-duration flashes was less than that of intermediate-duration flashes. Kitterle and Corwin (1979) called this effect "temporal contrast enhancement" and suggested that the changes in apparent contrast found with low-spatial-frequency targets reflect primarily activity of transient channels.

The purpose of this study was to provide, by determining the effects of flicker adaptation upon the apparent contrast of low-spatial-frequency targets, a test of the hypothesis proposed by Kitterle and Corwin (1979). Recently, Breitmeyer, Levi, and Harwerth (1981) have shown that adaptation to a flickering uniform field of $6 \mathrm{~Hz}$ has several effects on visual performance at threshold and suprathreshold levels of stimulation. As a result of flicker adaptation, reaction time to low-spatial-frequency gratings is increased, the interstimulus interval necessary for resolving two briefly flashed suprathreshold lowspatial-frequency gratings increases, and contrast sensitivity for the detection of low-spatial-frequency gratings is reduced. Breitmeyer et. al. (1981) suggested that the uniform field flicker primarily adapted transient channels. Thus, if transient channels mediate temporal contrast enhancement, flicker adaptation should diminish or abolish the effect.

\section{METHOD}

\footnotetext{
Observers

Thirty undergraduates participated in this experiment for credit in introductory psychology. All had normal or corrected-to-normal vision and were naive about the purpose of this experiment.

Apparatus

The stimuli were sinusoidal gratings generated by means of a two-channel hapliscopic system. Each channel consisted of an oscilloscope (Tektronix 5103/DN, P-31 phosphor) upon which the gratings were generated by conventional methods (Campbell \& Green, 1965). Both oscilloscopes were masked down to circular apertures that at a viewing distance of $167 \mathrm{~cm}$ subtended a visual
}

angle of $3.4 \mathrm{deg}$. The screens were spatially adjacent and separated by $2 \mathrm{deg}$. Midway between the display screens was a dim red fixation light. The luminance of both screens was carefully matched to $10 \mathrm{~cd} / \mathrm{m}^{2}$, and grating contrast was $34 \%$ as measured with an EG\&G photometer/radiometer. A Sym-1 microprocessor (Synertek Corp.) was used to control the timing and collection of data.

\section{Procedure}

The task of the subjects was to determine which of two briefly flashed sinusoidal gratings of $.67 \mathrm{cycles} / \mathrm{deg}$ had the greater contrast. Both of the targets had the same physical contrast, but, on a given trial, one target was presented for $T$ msec and the other for $500 \mathrm{msec}$. This technique is similar to that described by Bowen and Pokorny (1978). The data generated are plotted to show the percentage of time the longer $(500-\mathrm{msec})$ flash is judged to have a greater contrast as a function of the shorter ( $T$ msec) flash. At very short durations, the 500 -msec flash should always be judged to be greater in contrast than the shorter flash. If, however, there is a temporal contrast enhancement effect, there will be a duration $T$ for which the longer flash is never judged to have a greater contrast. Finally, at sufficiently long durations, the T-msec and 500 msec flashes should be judged not to differ in apparent contrast, and thus the judgments should asymptote around $50 \%$.

The 30 subjects were divided into three groups of 10 . There were two experimental groups, both of which were binocularly adapted. One of these groups adapted initially for $2 \mathrm{~min}$ to a stationary low-spatial-frequency sinusoidal grating of $.67 \mathrm{cycles} / \mathrm{deg}$. The other experimental group adapted to a 6-Hz flickering grating of $.67 \mathrm{cycles} / \mathrm{deg}$ for the same duration. The control group viewed a blank screen that was of the same mean luminance as the adapting gratings. After $500 \mathrm{msec}$, the adaptation condition was terminated and the two test gratings were presented. The subject pressed one of two keys to indicate which of the two gratings had the greater perceived contrast. Five hundred milliseconds after this judgment, the adapting condition was restored for $20 \mathrm{sec}$ and the cycle was repeated until 14 replications had been made at each $T$ duration.

\section{RESULTS}

The results of this experiment are shown in Figure 1 . The percentage of time that the $500-\mathrm{msec}$ flash is judged to have more contrast is plotted as a function of the duration of the shorter flash. The filled and open circles show the results obtained for the unadapted and stationary-adapted conditions, respectively. The vertical bars indicate the $95 \%$ confidence interval. The fact that the two curves are virtually indistinguishable and that both dip significantly below $50 \%$ within the range of $60-90 \mathrm{msec}$ indicates that the Broca-Sulzer effect is not abolished by stationary adaptation. However, the results obtained with flicker adaptation (plotted as filled triangles) show a monotonic decline with duration of the shorter pulse. There does not appear to be any duration for which the shorter pulse is seen to have more contrast than the 500-msec flash. This indicates that flicker adaptation has abolished temporal contrast enhancement.

\section{DISCUSSION}

Although temporal contrast enhancement is found only with low-spatial-frequency gratings (Kitterle \& 


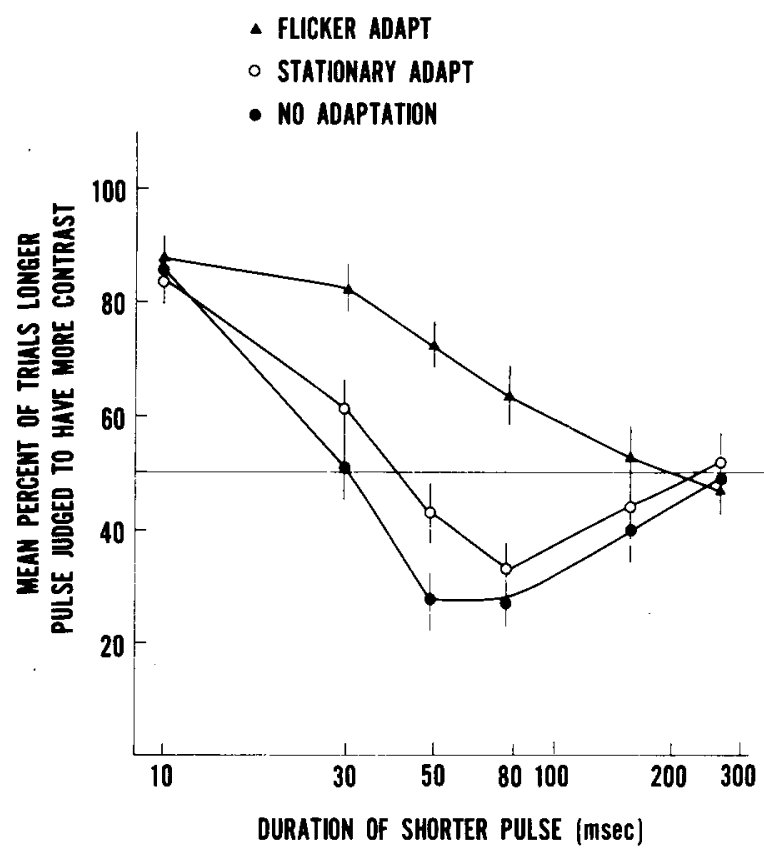

Figure 1. The percent of time the $500-\mathrm{msec}$ pulse was judged to have a greater contrast as a function of the duration of the shorter pulse under flicker adaptation (filled triangles), stationary adaptation (open circles), and no adaptation (filled circles). Each symbol represents the mean of $\mathbf{2 1 0}$ judgments. Vertical bars represent the $95 \%$ confidence interval.

Corwin, 1979; Kitterle \& Rysberg, 1976), it cannot be concluded that the effect is necessarily due to activity solely within transient channels. Transient and sustained channels overlap considerably in the range of spatial frequencies to which each responds. Consequently, at suprathreshold levels, sustained channel activity may jointly contribute to this effect. However, Breitmeyer et al. (1981) have shown that, at threshold and above threshold, flicker adaptation modifies only transient channel activity. Thus, flicker adaptation provides a means of testing the role of transient mechanisms in temporal brightness and temporal contrast enhancement. The present paper complements these earlier papers and provides strong support for the role of transient channel activity in temporal contrast enhancement. Flicker adaptation appears to have selectively reduced the contribution of transient channels to temporal changes in apparent contrast. It has been suggested that flicker adaptation or any other procedure that selectively reduces transient activity may shift processing to the more sensitive sustained mechanisms (Breitmeyer et al., 1981; Legge, 1978).

There are several features of the flicker-adapted curve (see Figure 1) that suggest that adaptation may have also switched the processing of this target to a different mechanism. First, as mentioned earlier, flicker adaptation abolishes temporal contrast en- hancement. This effect is not found for stimuli that activate primarily the sustained mechanism. Second, besides abolishing temporal contrast enhancement, flicker adaptation appears to shift the point at which the curve crosses the $50 \%$ line to a considerably longer duration (i.e., $300 \mathrm{msec}$ ), as opposed to $30-40 \mathrm{msec}$ for the other two conditions). Legge (1978) has shown that when transient channel activity is masked, the limits of temporal summation obtained with a lowspatial-frequency grating increases. Legge (1978) suggests that this result reflects a change in the mechanism that processes the grating (i.e., from transient to sustained). Thus, the present data obtained under flicker adaptation might reflect the temporal activity of the sustained mechanism. It is interesting to note that, in a recently completed study (Kitterle \& Corwin, 1983), it was found that over a range of target durations, the curve described by targets that were turned on gradually (i.e., with a rise time of $20 \mathrm{msec}$ ) did not show evidence of temporal contrast enhancement. Thus, ramping on a grating target abolishes temporal contrast enhancement. This procedure also reduces transient activity at both threshold (Breitmeyer \& Julesz, 1975) and at suprathreshold levels (Matsumura, 1976). Thus, it appears that experimental procedures that reduce the contribution of transient activity also reduce or abolish temporal contrast enhancement. This finding rather strongly suggests that transient activity is a condition necessary for obtaining contrast enhancement.

The present results may also have implications for understanding the mechanisms involved in temporal brightness enhancement. At high luminance levels, brightness enhancement is reduced or abolished (Aiba \& Stevens, 1964; Magnussen \& Bjorklund, 1979). In view of the differences in contrast gain of these two mechanisms, it is quite possible that at these luminance levels the transient response has saturated. The result would be a shift in the processing of brightness to sustained channels. Consistent with this hypothesis is the finding that, at very high luminance levels, the critical duration for the temporal summation of brightness increases (Aiba \& Stevens, 1964). It should not be concluded that sustained channels do not play a role in brightness. At long durations, apparent contrast is greater for square-wave gratings than for sinusoidal gratings of the same fundamental frequency (Kitterle \& Corwin, 1979). Since square-wave gratings contain higher harmonics, these findings suggest that sustained channels may code the brightness of long-duration targets. Although the candidate code for brightness is not known because it is not clear how the sensory response varies with time for a flash of a given duration, transient channels respond with an initial high burst of activity followed by lower maintained activity (Green, 1981 b; Mitov et al., 1981). This is not the case for sustained channel activity, in which the initial burst is not found. Thus, if this 
particular neural feature is important, any procedure that diminishes its magnitude should alter the brightness response. Consequently, it would be of interest to examine the influence of flicker adaptation upon brightness enhancement with uniform fields. The present results suggest that steady adapting fields should have little, if any, effect on temporal brightness enhancement, since they do not adapt transient channels. Consistent with this interpretation are the results of Aiba and Stevens (1964), who did not find any effect of steady adaptation on brightness enhancement. If flicker adaptation reduced brightness enhancement, then this technique could provide insight into the candidate code for brightness. In particular, these measures could test directly the hypothesis that the initial transient activity that accompanies the onset of a flash is a prerequisite for obtaining brightness enhancement. This finding would contradict those theories that ascribe little importance to the role of neural response transients to this phenomenon (Wasserman \& Kong, 1974).

\section{REFERENCES}

ADRIAN, E. D. The basis of sensation: Action of the sense organs. London: Christophers, 1928.

ArbA, T. S., \& Stzvens, S. S. Relation of brightness to duration and luminance under light- and dark-adaptation. Vision Research, 1964, 4, 391-404.

Baumandot, E., \& Szant, J. Facilitation et inhibition, parametres de ia fonction visuelle. Année Puychologique, 1947, 43-44, 54-102.

Bowen, R. W., \& Poxonvy, J. Target edge sharpness and temporal brightness enhancement. Vision Research, 1978, 15, 1411-1412.

Boynton, R. M. Some temporal factors in vision. In W. Rosenblith (Ed.), Sensory communication. New York: Wiley, 1961.

Bre itMEYer, B. Simple reaction time as a measure of transient and sustained channels. Vision Research, 1975, 15, 1411-1412.

BreItMeYer, B., \& JuLEsz, B. The role of on and off transients in determining the psychophysical spatial frequency response. Vision Research, 1975, 15, 411-415.

Bagitueyer, B., Levi, D. M., \&arwerth, R. S. Flicker masking in spatial vision. Vision Research, 1981, 21, 1387-1394.

BrocA, A., \& Suleser, P. La sensation luminise en fonction du temps. Journal de Physiologie et de Pathalogie Générale, 1903, 6, 55-68.

Burseck, C. A. Criterion-free pattern and flicker thresholds. Journal of the Optical Society of America, 1981, 71, 1343-1350.

Bunasck, C. A., \& Kelty, D. H. Contrast gain measurements and the transient/sustained dichotomy. Journal of the Optical Society of America, 1981, 71, 1335-1342.

Campberl, F. W., \& Green, D. G. Optical and retinal factors affecting visual resolution. Journal of Physiology, 1965, 181, 576-593.

Green, M. A. Psychophysical relationships among mechanisms sensitive to pattern, motion and flicker. Vision Research, 1981, 21, 971-984. (a)
Green, M. A. Spatial frequency effects in masking by light. $V i$ sion Research, 1981, 21, 861-866. (b)

KeEsEy, U. T. Flicker and pattern detection: A comparison of thresholds. Journal of the Optical Society of America, 1972, 62, 446-448.

KELLY, D. H. Theory of flicker and transient responses. I. Uniform fields. Journal of the Optical Society of America, 1971, 61, 537-546. (a)

KELLY, D. H. Theory of flicker and transient responses. II. Counterphase gratings. Journal of the Optical Society of America, 1971, 61, 632-640. (b)

KITTERLE, F. L. Reaction time measures of suprathreshold spatiotemporal interactions. Supplement to Investigative Ophthalmology and Visual Science, 1979, 18, 250.

Kitte rLe, F. L., \& Corwin, T. R. Enhancement of apparent contrast in flashed sinusoidal gratings. Vision Research, 1979, 19, 33-39.

KitTenle, F. L., \& Conwin, T. R. The effects of temporal waveform upon apparent contrast. Perception \& Psychophysics, $1983,33,72-74$.

Kitterle, F. L., \& Rysbera, J. A. The effect of exposure duration on the apparent contrast of sinusoidal gratings. Perception \& Psychophysics, 1976, 1, 335-338.

Kitterle, F. L., Conwin, T. R., \& Benta, J. Masking of sinusoidal targets by uniform fields of unequal duration. Journal of the Optical Society of America, 1979, 69, 1445.

Kulikowsmi, J. J., \& Tolhurst, D. J. Psychophysical evidence for sustained and transient detectors in human vision. Journal of Physiology, 1973, 232, 149-162.

LEOOE, G. E. Sustained and transient mechanisms in human vision: Temporal and spatial properties. Vision Research, 1978, 18, 69-81.

Maanussen, S., \& Bjorirund, R. A. The perception of suprathreshold sinusoidal flicker measured by light- and dark-phase matching. Vision Research, 1979, 19, 335-338.

Matgumura, M. Visual masking by luminance increment and decrement: Effects of rise time and decay time. Tohoku Psychologica Folia, 1976, 35, 104-114.

Mitov, D., VAssilev, A., \& ManaHilov, V. Transient and sustained masking. Perception \& Psychophysics, 1981, 30, 205-210.

NACHMiAB, J. The effect of exposure duration on visual contrast sensitivity with square-wave gratings. Journal of the Optical Society of America, 1967, 57, 421-427.

Tolhurst, D: J. Separate channels for the analysis of the shape and movement of a moving visual stimulus. Journal of Physiology, 1973, 231, 385-402.

UENo, T. Temporal characteristics of the human visual system as revealed by reaction time to double pulses of light. Vision Research, 1977, 17, 591-596.

van Neb, F. L., Koenderink, J. J., Na8, H., \& Bouman, M. A. Spatiotemporal modulation transfer in the human eye. Journal of the Optical Society of America, 1967, 57, 1082-1088.

WAsernuAN, G. L., \& Kona, K. L. Illusory correlation of brightness enhancement and transients in the nervous system. Science, 1974, 184, 911-913.

Watson, A. B., \& Nachuias, J. Patterns of temporal interaction in the detection of gratings. Vision Research, 1977, 17, 893-902.

(Manuscript received June 1, 1982;

revision accepted for publication October 20, 1982.) 